\title{
Sharing Location Information in Multi-UAV Systems by Common Channel Multi-Token Circulation Method in FANETs
}

\author{
Eyup Emre Ulku', Buket Dogan ${ }^{1}$, Onder Demir ${ }^{1}$, Ilker Bekmezci ${ }^{2}$ \\ ${ }^{I}$ Department of Computer Engineering, Marmara University Faculty of Technology, \\ Goztepe Campus 34722/Kadikoy - Istanbul, Turkey \\ ${ }^{2}$ Independent Scholar \\ emre.ulku@marmara.edu.tr
}

\begin{abstract}
Unmanned Aerial Vehicle (UAV) technology is being used increasingly for military and civilian purposes. The primary reason for this increase is that UAVs eliminate the risk to human life in difficult and dangerous missions, are cost effective, and easily are deployed. Developments in UAV technology and decreasing costs have increased UAV usage. However, when multiple UAVs are deployed, inter UAV communication becomes complicated. For this reason, communication in multi-UAV systems is the most important problem that needs to be solved. To enable communication among UAVs without infrastructure support, a Flying Ad Hoc Network (FANET) is used. A FANET provides UAVs to fly in tandem without colliding. To ensure coordinated flight, UAVs require the location information of other UAVs. In this study, we developed a common channel multi-token circulation protocol to share location information in multi-UAV systems that communicate using a FANET. The proposed method ensures that UAVs in multi-UAV systems know each other's coordinate information with minimum error.
\end{abstract}

Index Terms-Flying Ad Hoc networks (FANET); Mobile Ad Hoc networks; Multi-token circulation; Unmanned aerial vehicle (UAV).

\section{INTRODUCTION}

Autonomous vehicles are capable of making decisions and do not require a driver or pilot [1]. Unmanned Aerial Vehicles (UAV) are a type of autonomous vehicle commonly used for military and civilian purposes [2]. The applications of UAVs are constantly increasing. For example, UAVs are used for search and rescue operations [3]-[5], border tracking [6], observation [7]-[9], monitoring [10], [11], reconnaissance [12], target tracking [13], disaster management [13] and creating large-scale maps [14]. Completing difficult missions without endangering human life is the most important reason for this increase [15], [16]. In addition, developments in automation and detection technologies, and decreased costs have also increased the use of UAVs [17].

With decreasing costs and advances in miniaturization technology, UAV systems comprising multiple mini UAVs have been realized [17], [18]. Such systems are referred to as UAV groups or teams. Recently, with the increase in usage of mini and micro UAVs, UAV swarms comprising a

Manuscript received 2 March, 2018; accepted 3 November, 2018. large number of mini- or micro-UAVs are becoming more common [19], [20]. With multi-UAV systems, complicated and difficult missions can be completed efficiently in much less time. In multi-UAV systems the level of autonomy and protocols to enable communication between UAVs have become more complicated. Thus, methods to facilitate multi-UAV communication are required [21].

To establish communication and coordination between UAVs, infrastructure equipment such as a ground station as shown in Fig. 1, or a satellite, is commonly used [22]. However, in these structures communication is limited to the area provided by the infrastructure. Therefore, communication between UAVs is limited to the coverage area of the satellite or ground station. In addition, UAVs cannot be controlled if the infrastructure does not provide communication. In addition to this, other problems also arise in infrastructure based methods. For instance, each UAV requires expensive and complex equipment to communicate with the infrastructure. Communication security poses another problem. UAV communication links may break due to dynamic environmental conditions, node movement, and geographical features. For example, obstacles such as mountains, walls, and buildings can block communication signals [15], [17], [18].

A Flying Ad Hoc Network (FANET) is an alternative solution to establish communication between UAVs without an infrastructure.

In the literature, FANET is the name of the ad hoc network that is established between UAVs. A FANET model is shown as an example in Fig. 2.

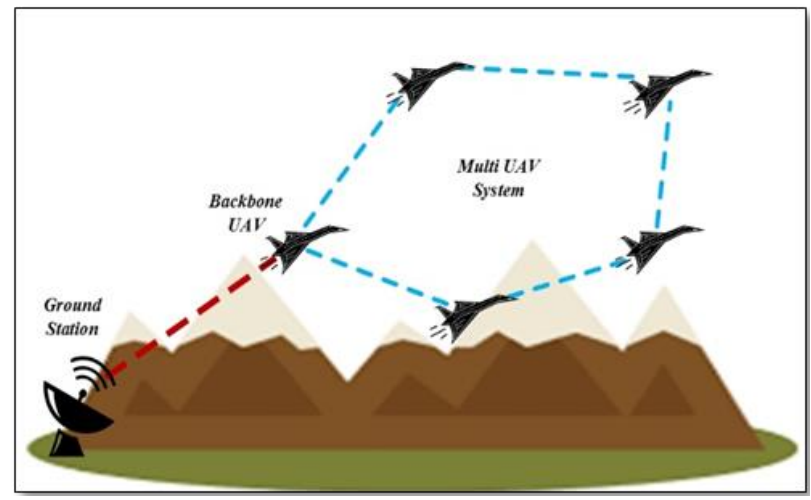

Fig. 1. Ground station-based communication. 


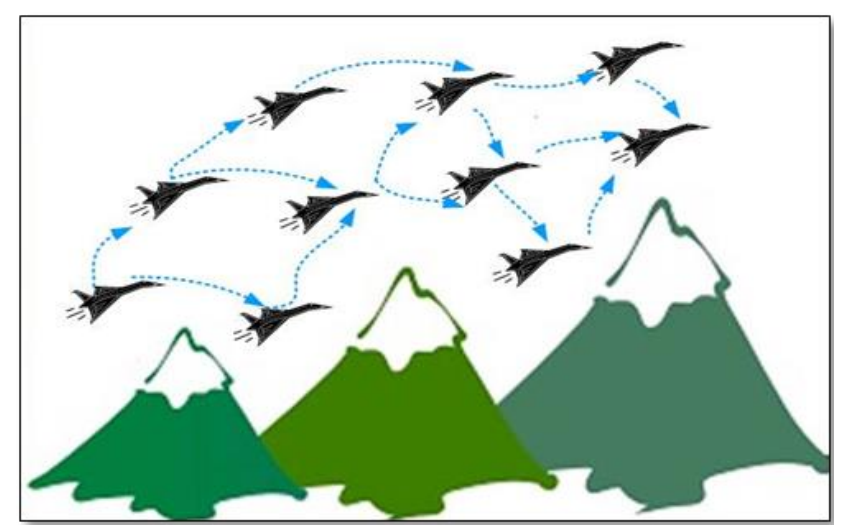

Fig. 2. Flying Ad Hoc network.

A FANET is an effective solution for communication problems that occur in multi-UAV systems; however, for safe flight UAVs in a FANET must know each other's location information. To ensure that UAVs possess this location information, a laser based imaging Light Detection and Ranging (LIDAR) system that is shown in Fig. 3 can be used [23]. However, LIDAR equipment is too heavy for use with mini- or micro-UAVs and LIDAR systems consume significant energy, which reduce UAV flight time. In addition, LIDAR equipment is expensive. Therefore, an alternative solution is required to ensure that UAVs in a FANET can obtain the required location information.

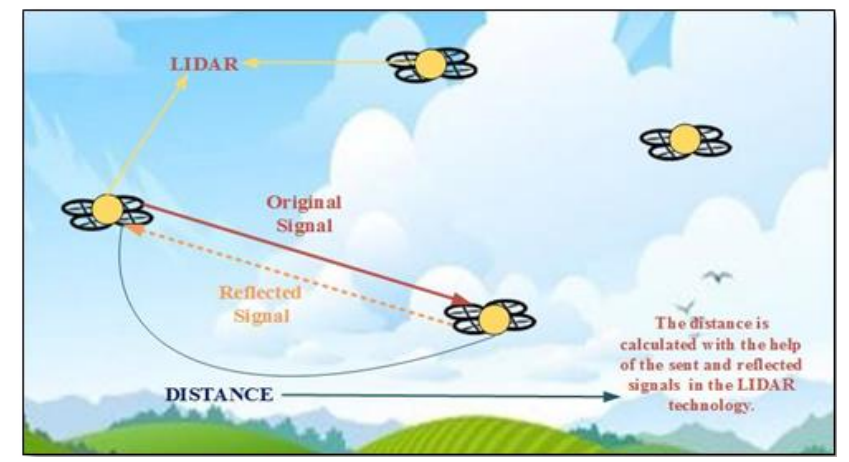

Fig. 3. LIDAR technology.

A token circulation-based approach is an alternative solution for UAVs to learn each other's coordinates. In this approach, a token package that holds coordinate information of UAVs circulates in the FANET. As the number of UAVs in the FANET increases, the size of the data in the token package and the number of nodes that the token must reach increase. Thus, the duration of token travel time increases. Consequently, UAVs may not receive accurate location information. To avoid this, travel duration is reduced using multiple tokens. Therefore, UAVs know each other's location information with fewer error. In previous tokenbased studies, it was assumed that each token uses a different channel and that UAVs support such a communication structure [15], [17], [18]. However, in this study, tokens circulate through a common single channel. In this structure, the collision problem of the tokens arises. A second channel is used to minimize token collisions. This two-channel multi-token based approach allows location information to circulate in multi-UAV systems.

The remainder of this paper is organized as follows. In Section II, a multi-token circulation system with a common single channel that enables UAVs in multi-UAV systems to obtain the location information of other UAVs in a FANET is explained. In Section III, performance results are presented, and, in Section IV, conclusions and suggestions for future work are given.

\section{COMmon CHANNEl Multi-TOKEn CirCulation}

Multi-UAVs within the FANET require each other's coordinate information to make a trouble free and coordinated flight. In this section, the common channel multi-token circulation model that is developed to ensure the circulation of location information between UAVs in a FANET, is explained.

The locations of highly mobile UAVs in a FANET change rapidly, and a token-based approach can enable UAVs to know the location information of other UAVs in the network. However, as the number of UAVs in the FANET increases, the size of the package that carries the location information and the number of the UAVs the package must reach also increase. Consequently, the duration of the token's travel time between each UAV also increases; thus, the UAVs can receive inaccurate location information. In such a situation, safe, coordinated flight would not be possible. Note that a single token is insufficient in multi-UAV systems if the number of UAVs exceeds a threshold. Therefore, increasing the number of tokens traveling in the FANET optimally, in parallel with the number of UAVs in FANET, constitutes the basis for multi-token structure.

Increasing the number of tokens enables UAVs to receive more accurate location information. However, increasing the number of tokens may result in lost, delayed, or incorrect information due to token collisions. Previous studies, employed separate channels to prevent collision in multitoken systems [15], [17], [18].

We developed two-channel model for multi-token circulation that is independent of the number of tokens. In this model, tokens circulate in a single common channel and are routed via a second channel such that collisions are avoided.

The structure of the tokens with the UAV location information is shown in Fig. 4. As shown, a token comprises four parts, i.e., a unique token number, the source address, the destination address, and the location information of each UAV in the FANET. First of them is the area for token number. Because there is more than one token circulating in FANET, the unique value that is used for identifying the tokens are placed in that area. In the second area, there is a source address section that will enable the UAVs to find out from which UAV the token has come. The third area consists of the destination address that indicates to which UAV the token should be sent, and in the last area there is location information of every UAV in FANET.

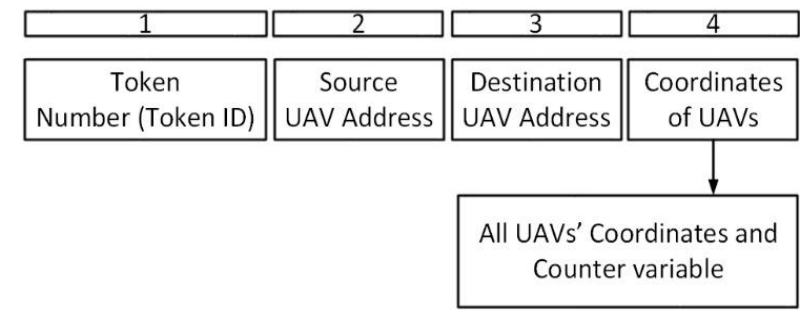

Fig. 4. Token format. 
A flow chart of the structure that enables tokens to travel between UAVs in FANET, is shown in Fig. 5. As can be seen, when a token is traveling in FANET, the UAV that receives the token will add its coordinate information. In addition, it will compare the data in the token to the data in its memory to update either its memory or the token with the most current information. A counter variable is used for this comparison operation. As the position information changes, the value of the counter variable increases, such that the coordinate with the highest counter value represents the most current location information.

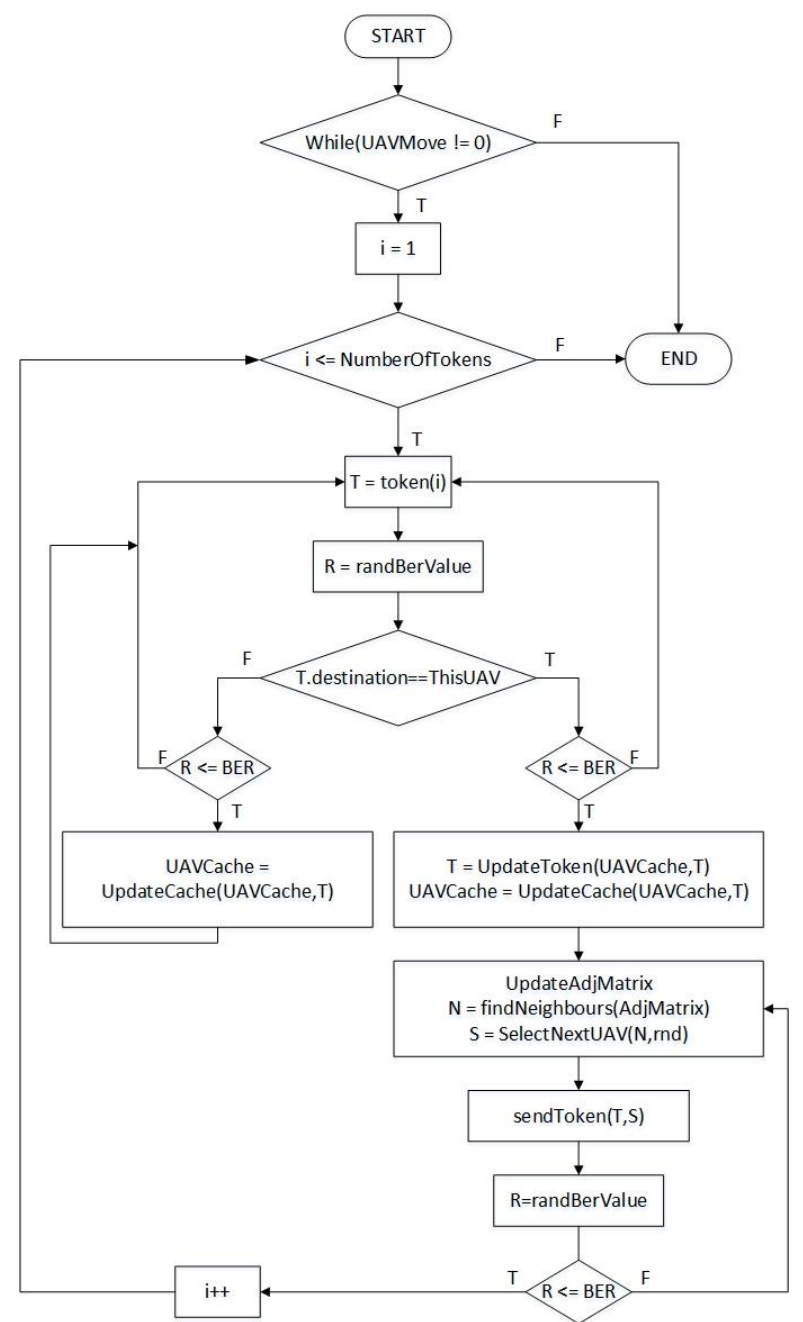

Fig. 5. Flowchart of the proposed multi-token circulation method in a FANET.

The concept of "overhearing" is also considered in the proposed method. Non-destination UAVs can overhear a sent token. Note that undirected antennas are generally used in wireless communication. With undirected antennas, the signal is propagated to all sides, which results in the information being transmitted to the target's neighbours. This situation can be considered an advantage. As shown in Fig. 6, neighbouring UAVs overhear a token even if they are not the target, and these UAVs will receive and store the information if it is more current than the information they currently possess. However, if the information they already possess is more current, they cannot change it because they do not possess the token.

The bit error rate (BER) is an important factor that affects performance. BER is the proportion of corrupted or incorrect bits detected in the transmission of digital data
[24]. We must ensure that the package sent from one UAV to another in a FANET is delivered correctly without problems. If the package fails to reach the destination or if it arrives with corrupted data, it must be resent. A bit error check is performed for each packet. The Rician fading model is used to perform a bit error check to calculate the BER. The probability density function (pdf) of Rician distribution is shown in (1) [25]. In this formula, $A$ symbolises the peak amplitude of dominant signal, $\sigma$ denotes the root mean square ( $\mathrm{rms}$ ) value of the received signal and $r$ is the amplitude of the received signal envelope. In addition to these, the modified Bessel function of the first kind and zero order is representing by $\mathrm{I}_{0}$. The Rician distribution is generally defined as the ratio between the power of the dominant signal and the variance of the multipath components. This expression is defined by the $K$ parameter when it is called the Rician factor as shown in (2) [26]. With the help of Rician model, BER is calculated as shown in (3). $E_{b} / N_{0}$ denotes the energy per symbol to noise power spectral density ratio. Then, the BER value is used as the threshold value in packet transmission. The BER is calculated for all sent and overheard packets:

$$
p d f(r)=\frac{r}{\sigma^{2}} e^{\left(\frac{r^{2}+A^{2}}{2 \sigma^{2}}\right)} I_{0}\left(\frac{r . A}{\sigma^{2}}\right)
$$

where $r \geq 0, A \geq 0$.

$$
\begin{gathered}
K[d B]=10 . \log \left(\frac{A^{2}}{2 \sigma^{2}}\right), \\
P_{b}=\frac{1}{2} \operatorname{erfc}\left\lfloor\sqrt{\frac{K\left(E_{b} / N_{0}\right)}{K+E_{b} / N_{0}}}\right\rfloor .
\end{gathered}
$$

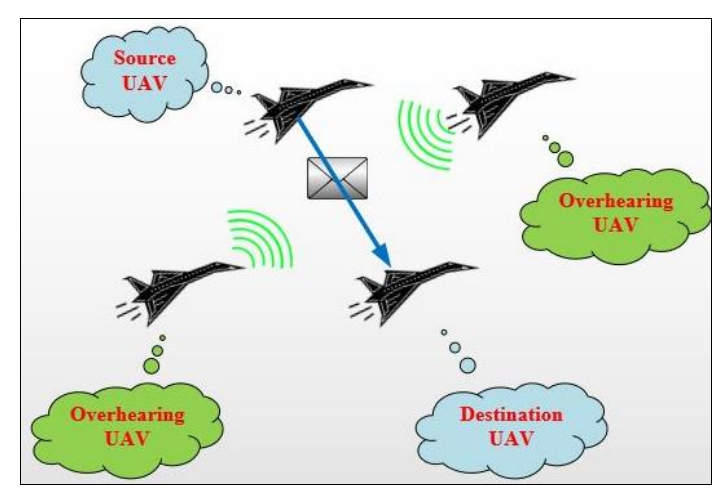

Fig. 6. Overhearing UAVs.

Packet transmission time $\left(\mathrm{P}_{\mathrm{TT}}\right)$ is one of the most important parameters to determine system performance in the proposed token-based approach. In communication systems, transmission time is defined as the elapsed time between the start of sending a token and the arrival of the last bit of the token at the target node [27]. Two basic parameters are used to calculate the transmission time, i.e., packet size and bit transmission rate, as shown in (4). To make the analysis of the latency time in the network more understandable, the packet delay $\left(\mathrm{D}_{\mathrm{T}}\right)$ is divided into a sequence of node delays. The equation (5) shows the formulation of the delay analysis. The time that a node 
spends processing a packet is called a processing delay $\left(D_{\text {proc }}\right)$ that can be neglected as it is considerably smaller than the other delays in the equation. The time it takes to put the entire packet in the communication media is called the transmission delay ( $\left.\mathrm{D}_{\text {trans }}\right)$. The equation (6) shows the calculation of the transmission delay. Here, the transmission speed is denoted $R$ and the length of a packet in bits are denoted by $L$. Another delay parameter is the queuing delay $\left(D_{\text {queue }}\right)$. Queuing delay is a latency that the package is spent waiting on a node. Here, $1_{\text {queue }}$ is the average length of the queue. In the communication media, the required signal change to travel from one node to another is called propagation delay $\left(\mathrm{D}_{\text {prop }}\right)$. The propagation delay is calculated as shown in (8). The parameters used in the formula are the distance (D) from one node to the other node and the propagation speed of the medium, which is a constant value used to calculate the transmission delay (s) [28], [29]. As shown in (9), packet delay $\left(\mathrm{D}_{\mathrm{T}}\right)$ is added to the packet transmission time obtained by (4), and the packet delivery time $\left(\mathrm{P}_{\mathrm{DT}}\right)$ is calculated:

$$
\begin{gathered}
P_{T T}=\frac{\text { PacketSize }}{\text { BitRate }}, \\
D_{T}=D_{\text {proc }}+D_{\text {queue }}+D_{\text {trans }}+D_{\text {prop }}, \\
D_{\text {trans }}=L / R, \\
D_{\text {queue }}=D_{\text {trans }} \times I_{\text {queue }}, \\
D_{\text {prop }}=\frac{D}{s}=\left(\frac{\text { Communication Range }}{3 \times 10^{8}}\right), \\
P_{D T}=P_{T T}+D_{T} .
\end{gathered}
$$

While token circulation occurs in the first channel, small talk packages circulating in the second channel prevent the tokens from colliding. In the second channel, three different packages are used, i.e., Clear to Send (CTS), Request to Send (RTS) and Not Clear to Send (NCTS) packages. A flowchart of the algorithm developed to minimize token collision is shown in Fig. 7. The token holder UAVs determine the destination neighbours to send tokens to according to the talk packages in the second channel. First, neighbours are determined using the adjacency matrix of the UAV holding the token. In this phase, neighbours are eliminated by means of the talk packages in the second channel and the proposed method attempts to find the best target UAV. Neighbours sending CTS, RTS and NCTS packages that are overheard by the token holder UAV are eliminated before sending the token to avoid collisions. If the UAV has overheard that a neighbour has sent a CTS package, it eliminates this neighbour because it is taking another token. When a UAV is emitting an RTS or NCTS package it cannot receive another token. After this elimination, the number of neighbours is checked, and, if only one neighbour remains, the token is sent to this neighbour. If there are no neighbours, a token is hold as long as the time of token sending. If there is more than one neighbours after the elimination process and the source UAV from which the token is received is also in the list, this source UAV is eliminated to ensure that the information in the entire network is current by routing tokens in different directions.

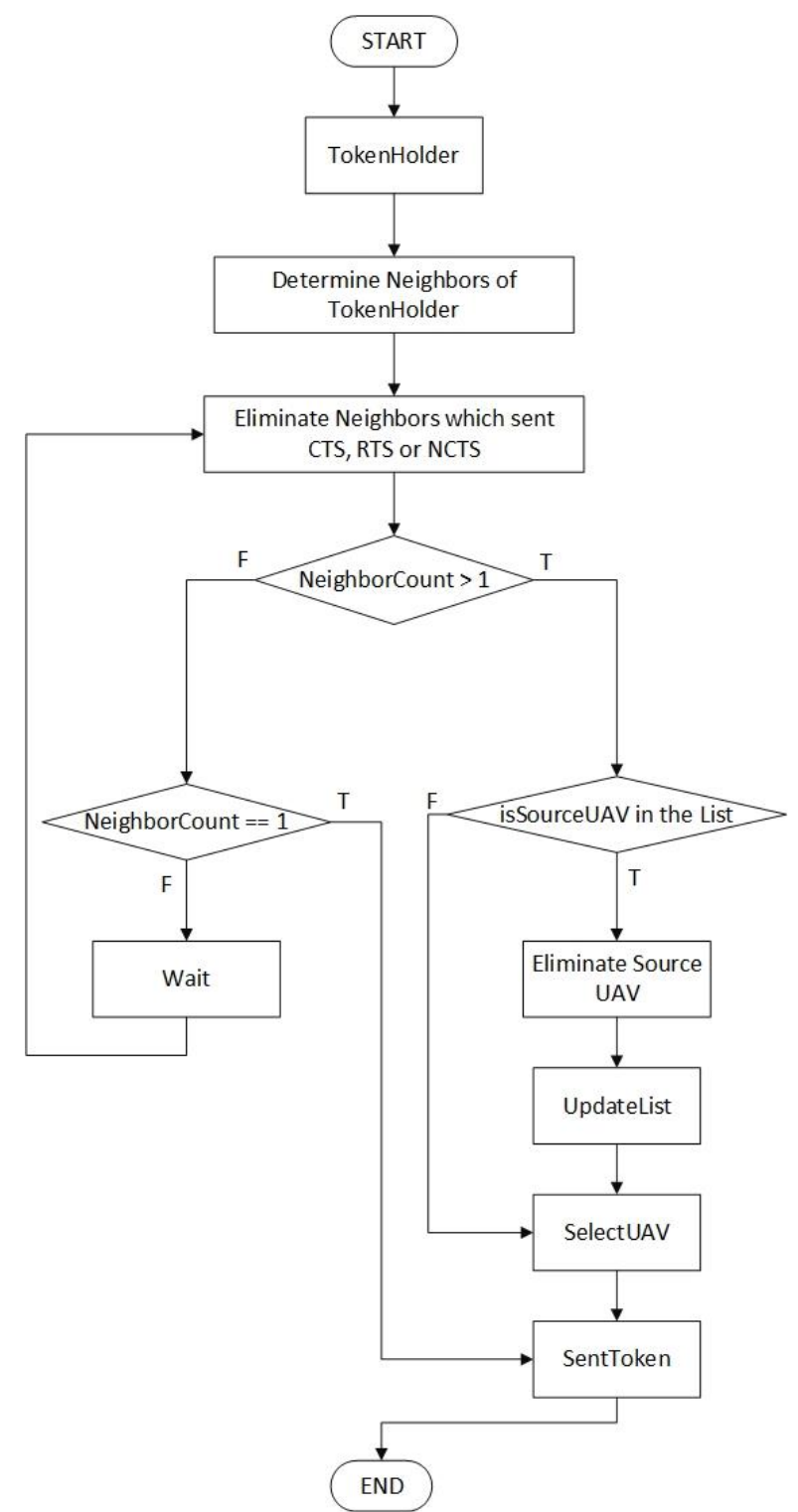

Fig. 7. Flowchart of the algorithm to avoid collisions.

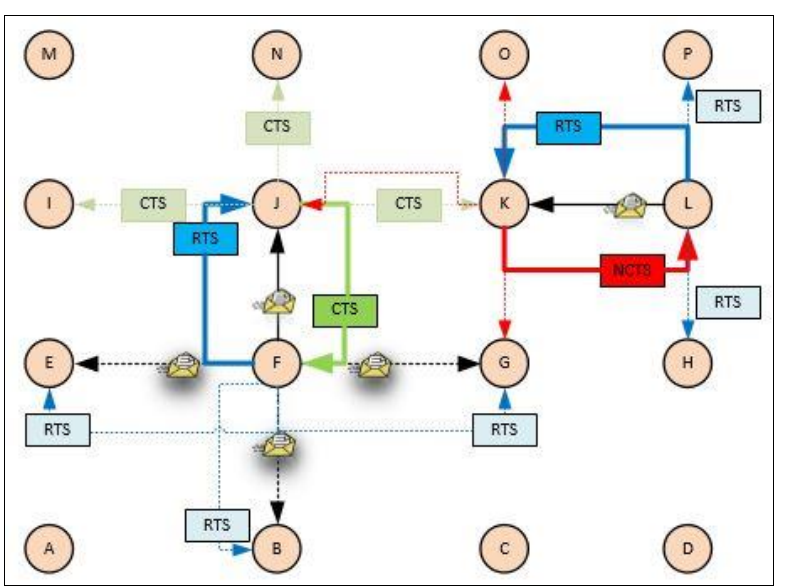

Fig. 8. Example scenario of the proposed two-channel multi-token circulation model.

An example scenario of the proposed two-channel multitoken circulation model is shown in Fig. 8. Here, K knows it will obtain or overhear the token in the next step because it heard the CTS package. Thus, it sends an NCTS package to $\mathrm{L}$ who wants to send it a token. This avoids a possible collision in the next step. At this point, tokens circulate in the FANET and avoid collisions. 


\section{Performance Results}

The parameters used in our simulation environment are shown in Table I. The simulation results obtained with these parameters are shown in Fig. 9.

TABLE I. SIMULATION PARAMETERS AND VALUES.

\begin{tabular}{|c|c|}
\hline Parameter & Values \\
\hline Number of UAVs & $10,20,30,40,50$ \\
\hline Number of Tokens & $1,2,3,4,5,6$ \\
\hline UAVs Speed & $5,7 \mathrm{u} / \mathrm{s}$ (unit/second) \\
\hline Flight Topology & Grid Topology \\
\hline BER & $10-3,10-4,10-5,10-6,10-7$ \\
\hline Communication Range & $5 \mathrm{u}$ (unit) \\
\hline Channel Transmission Speed & $11 \mathrm{Mbps}$ (Megabit per second) \\
\hline Duration of Simulation & $60 \mathrm{~s}$ (second) \\
\hline Channel Type & Channel/Wireless Channel \\
\hline MAC Type & Mac/802.11 \\
\hline
\end{tabular}

The average error results obtained with varying numbers of UAVs and tokens for UAV speeds of $5 \mathrm{u} / \mathrm{s}$ and $7 \mathrm{u} / \mathrm{s}$ are shown in Fig. 9(a) and Fig. 9(b), respectively. As can be seen, the BER value is $10^{-5}$ and the communication range is $5 \mathrm{u}$. When the number of UAVs is greater than 20 , the effect of multi-token circulation is clearer. Thus, by increasing the number of tokens relative to the number of UAVs, the UAVs can learn location information with fewer errors. Note that as the UAV speed increases, the error rate and the effect of multi-token circulation also increase.

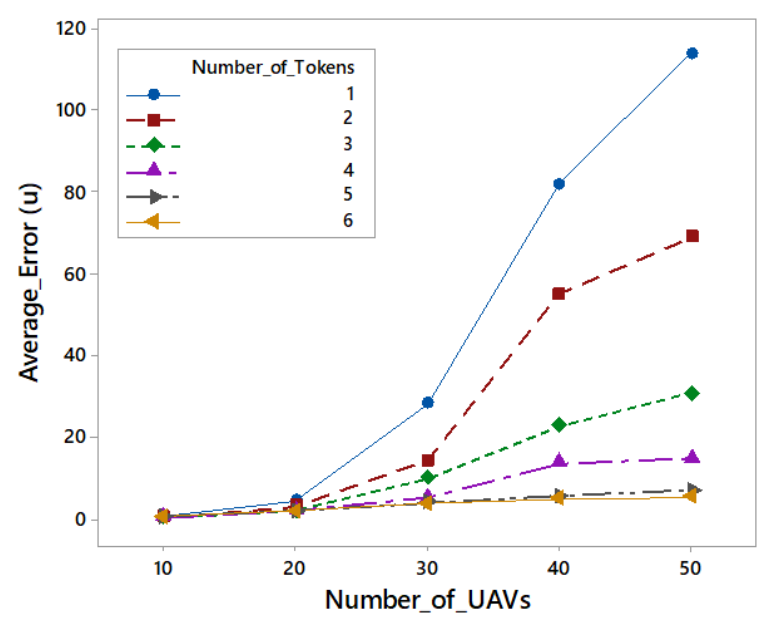

(a)

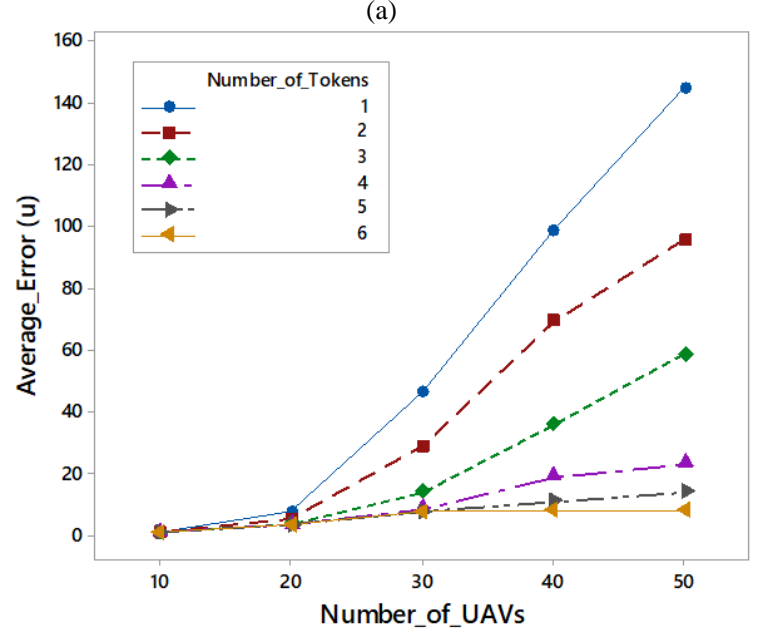

(b)

Fig. 7. Average location information error: a) UAV speed: $5 \mathrm{u} / \mathrm{s}$; b) UAV speed: $7 \mathrm{u} / \mathrm{s}$.

To ensure safe flight, UAVs should know each other's position with minimum error. However, the error rate can increase due to various conditions. For example, some tokens cannot be transmitted due to collisions. In addition, incorrectly transmitted tokens should be considered. Such tokens cause repeated sending. In such cases, the error rate can become very high. Figure 10 shows how retransmissions of tokens and collisions affect the average error rate. Here, the number of UAVs is 30 and the UAV speed is $5 \mathrm{u} / \mathrm{s}$. This graph shows how different BER values affect the results.

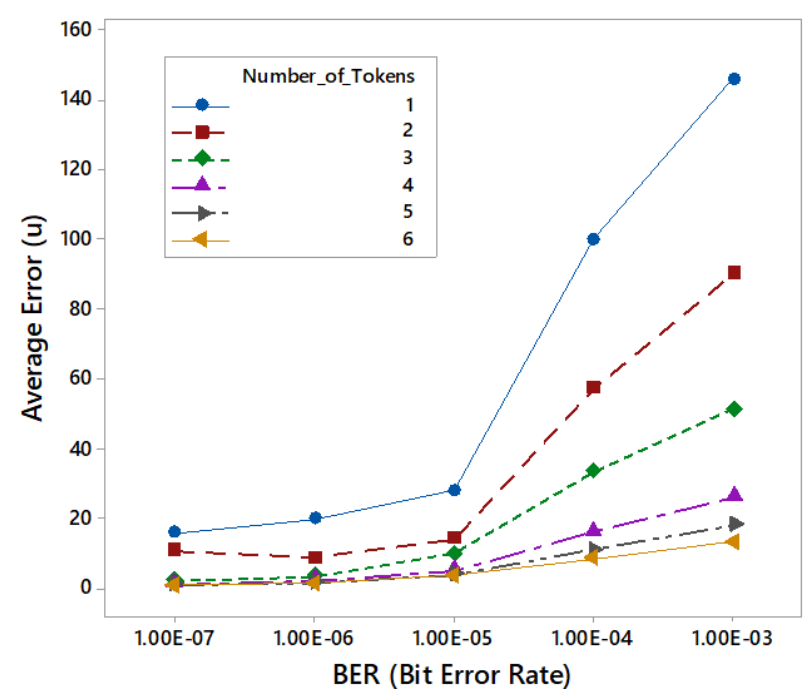

Fig. 8. Effects of BER on average error.

When the studies in the literature are examined, there are very few studies that use the token circulation method for UAV communication in the FANET. In previous studies, token circulation was used to provide code assignment for channel access [22], [30]. In these studies, code information that is much smaller than the location information is carried in the token. Since a small amount of information was carried within the token, the use of a single token was sufficient [22], [30]. In the developed multi-token circulation-based method, location information is carried in the tokens and as the number of UAV increases, the size of the information carried is also increasing. For this reason, we developed a multi-token-based method to circulate location information in the FANET. Thus, we have proposed a method that can be used outside the GPS and LIDAR technologies to learn position information of UAVs in the FANET.

\section{CONCLUSIONS}

Multi-UAV systems have been developed to increase the likelihood of success in high-risk tasks. With multi-UAV systems, difficult tasks can be completed safely in less time. In addition, communication between UAVs in multi-UAV systems is an important issue. In situations where it is impossible to provide a communication infrastructure, the FANET structure plays an active role in providing communication. After the problem of communication with FANET is solved, there is a need for a structure to exchange location information in FANET. To realize coordinated flights in multi-UAV systems, UAVs must be able to obtain the location information of other UAVs in the network.

In this paper, we have presented a new method that enables UAVs to learn each other's position information in the FANET due to multi-token circulation. 
In the proposed method, tokens carrying location information of UAVs circulate in a single common channel in the network and a second channel is used to prevent circulating tokens from colliding. Thus, with the two channel structure collisions in multi token circulation are avoided.

In future, we plan to extend the results obtained in this study to the GNU Radio platform. In addition, the results obtained in the simulation environment can be realized practically using software-based radio technology.

\section{REFERENCES}

[1] H. Hastie, X. Liu, Y. Petillot, P. Patron, "Talking autonomous vehicles: Automatic AUV mission analysis in natural language", in OCEANS 2017 - Aberdeen, 2017, pp. 1-5. DOI: 10.1109/OCEANSE.2017.8084617.

[2] O. Bouachir, A. Abrassart, F. Garcia, N. Larrieu, "A mobility model for UAV ad hoc network", in Int. Conf. Unmanned Aircraft Systems (ICUAS 2014), Orlando, FL, USA, 2014, pp. 383-388. DOI: 10.1109/ICUAS.2014.6842277.

[3] D. Erdos, A. Erdos, S. E. Watkins, "An experimental UAV system for search and rescue challenge", IEEE Aerospace and Electronic Systems Magazine, vol. 28, no. 5, pp. 32-37, 2013. DOI: 10.1109/MAES.2013.6516147.

[4] M. A. Goodrich, L. Lin, B. S. Morse, "Using camera-equipped miniUAVS to support collaborative wilderness search and rescue teams", in Int. Conf. Collaboration Technologies and Systems (CTS 2012), 2012, pp. 638-638. DOI: 10.1109/CTS.2012.6261008.

[5] P. Gaynor, D. Coore, "Towards distributed wilderness search using a reliable distributed storage device built from a swarm of miniature UAVs", in 2014 Int. Conf. Unmanned Aircraft Systems (ICUAS 2014), Orlando, FL, USA, 2014, pp. 596-601. DOI: 10.1109/ICUAS.2014.6842302.

[6] S. Berrahal, J. H. Kim, S. Rekhis, N. Boudriga, D. Wilkins, J. Acevedo, "Border surveillance monitoring using quadcopter UAVaided wireless sensor networks", Journal of Communications Software and Systems, vol. 12, no. 1, pp. 67-82, 2016. DOI: 10.24138/jcomss.v12i1.92.

[7] M. Abdelkader, M. Shaqura, G. C. Christian, W. Gueaieb, "A UAV based system for real time flash flood monitoring in desert environments using lagrangian microsensors", Int. Conf. Unmanned Aircraft Systems (ICUAS 2013), Grand Hyatt Atlanta, Atlanta, GA, 2013. DOI: 10.1109/ICUAS.2013.6564670.

[8] L. E. Caraballo, J. J. Acevedo, J. M. Diaz-Banez, B. C. Arrue, I. Maza A. Ollero, "The block-sharing strategy for area monitoring missions using a decentralized multi-UAV system", Int. Conf. Unmanned Aircraft Systems (ICUAS 2014), Orlando, FL, USA, 2014.

[9] K. Uto, H. Seki, G. Saito, Y. Kosugi, "Development of UAVmounted miniaturure hyperspectral sensor system for agricultural monitoring", Geoscience and Remote Sensing Symposium (IGARSS 2013), Melbourne, Australia, 2013. DOI: 10.1109/IGARSS.2013.6723814.

[10] L. Chen, Y. Jiang, C. Wang, "Adaptive visual servo control of UAV ground-target-autonomous-tracking system", 10th World Congress on Intelligent Control and Automation (WCICA 2012), Beijing, China, 2012. DOI: 10.1109/WCICA.2012.6357854.

[11] S. Lange, P. Protzel, "Cost-efficient mono-camera tracking system for a multirotor UAV aimed for hardware-in-the-loop experiments", 9th Int. Multi-Conf. Systems, Signals and Devices (SSD 2012), Chemnitz, Germany, 2012. DOI: 10.1109/SSD.2012.6198047.

[12] C. Kong, A. Ferworn, J. Tran, S. Herman, E. Coleshill, K. G. Derpanis, "Toward the automatic detection of access holes in disaster rubble", IEEE Int. Symposium on Safety, Security, and Rescue Robotics (SSRR 2013), Linkoping, Sweden, 2013. DOI: 10.1109/SSRR.2013.6719364.

[13] S. Gade, A. Joshi, "Heterogeneous UAV swarm system for target search in adversarial environment", Int. Conf. Control
Communication and Computing (ICCC 2013), Thiruvananthapuram, India, 2013. DOI: 10.1109/ICCC.2013.6731679.

[14] S. Siebert, J. Teizer, "Mobile 3D mapping for surveying earthwork projects using an Unmanned Aerial Vehicle (UAV) system", Automation in Construction, vol. 41, pp. 1-14, 2014. DOI: 10.1016/j.autcon.2014.01.004.

[15] I. Bekmezci, E. E. Ulku, "Location information sharing with multi token circulation in flying ad hoc networks", 7th Int. Conf. Recent Advances in Space Technologies (RAST 2015), Istanbul, Turkey, 2015. DOI: 10.1109/RAST.2015.7208427.

[16] D. Ho, E. I. Grotli, P. B. Sujit, T. A. Johansen, J. B. Sousa, "Clusterbased communication topology selection and UAV path planning in wireless sensor networks", Int. Conf. Unmanned Aircraft Systems (ICUAS 2013), Grand Hyatt Atlanta, Atlanta, GA, 2013. DOI: 10.1109/ICUAS.2013.6564674.

[17] E. E. Ulku, I. Bekmezci, "Multi token based location sharing for multi UAV systems", International Journal of Computer and Electrical Engineering, vol. 8, no. 2, pp. 197-206, 2016. DOI: 10.17706/ijcee.2016.8.3.197-206.

[18] E. E. Ulku, I. Bekmezci, "Multi token circulation in a common channel for location information sharing in multi-UAV systems", Int. Conf. Engineering and Natural Science (ICENS 2016), Sarajevo, Bosnia and Herzegovina, 2016. DOI: 10.17706/IJCEE.2016.8.3.197206.

[19] D. Shiyou, Z. Xiaoping, L. Guoqing, "Cooperative planning method for swarm uavs based on hierarchical strategy", 3rd Int. Conf. System Science, Engineering Design and Manufacturing Informatization, Chengdu, China, 2012. DOI: 10.1109/ICSSEM.2012.6340870.

[20] P. Bouvry, S. Chaumette, G. Danoy, G. Guerrini, G. Jurquet, "Using heterogeneous multilevel swarms of UAVs and high-level data fusion to support situation management in surveillance scenarios", IEEE lnt. Conf. Multisensor Fusion and Integration for Intelligent Systems (MFI 2016), Kongresshaus Baden-Baden, Germany, 2016. DOI: 10.1109/MFI.2016.7849525.

[21] I. Bekmezci, O. K. Sahingoz, S. Temel, "Flying ad-hoc networks (FANETS): a survey", Ad Hoc Networks, vol. 11, no. 3, pp. 12541270, 2013. DOI: 10.1016/j.adhoc.2012.12.004.

[22] J. Li, Z. Wei, Y. Zhou, M. Deziel, L. Lamont, F. R. Yu, "A tokenbased connectivity update scheme for unmanned aerial vehicle ad hoc networks", IEEE Int. Conf. Communications (ICC 2012), Ottawa, ON, Canada, 2012. DOI: 10.1109/ICC.2012.6363645.

[23] Y. Lin, J. Hyyppa, A. Jaakkola, "Mini-UAV-borne lidar for fine-scale mapping", IEEE Geoscience and Remote Sensing Letters, vol. 8, no. 3, pp. 426-430, 2011. DOI: 10.1109/LGRS.2010.2079913.

[24] N. Kostov, "Mobile radio channels modeling in MATLAB", Radioengineering, vol. 12, no. 4, pp. 12-16, 2003.

[25] S. Kumar, P. K. Gupta, G. Singh, D. S. Chauhan, "Performance analysis of Rayleigh and Rician fading channel models using Matlab simulation", I. J. Intelligent Systems and Applications, vol. 3, pp. 94$102,2013$.

[26] A. Tkac, V. Wieser, S. Pollak, "Calculation of impulse response in Rician and Rayleigh channel", Elektro 2012, Rajeck Teplice, Slovakia, 2012. DOI: 10.1109/ELEKTRO.2012.6225580.

[27] B. Krupanek, R. Bogacz, "Mathematical model of communication delays in wireless networks", INTL Journal of Electronics and Telecommunications, vol. 62, no.1, pp. 61-64, 2016. DOI: 10.1515/eletel-2016-0008

[28] S. Kakad, P. Sarode, J. W. Bakal, “A survey on query response time optimization approaches for reliable data communication in wireless sensor network", International Journal of Wireless Communications and Networking Technologies, vol. 1, no. 2, pp. 31-36, 2012.

[29] V. Markevičius, D. Navikas, D. Andriukaitis, M. Čepėnas, A. Valinevičius, M. Žilys, R. Malekian, A. Janeliauskas, W. Walendziuk, A. Idzkowski, "Two thermocouples low power wireless sensors network", AEU-International journal of electronics and communications, vol. 84, pp. 242-250, 2017. DOI: 10.1016/j.aeue.2017.11.032.

[30] J. Li, Y. Zhou, L. Lamont, M. Deziel, “A token circulation scheme for code assignment and cooperative transmission scheduling in CDMAbased UAV ad hoc networks", Wireless Network, vol. 19, pp. 14691484, 2013. DOI: 10.1007/s11276-013-0545-5. 